\title{
PROCESSAMENTO DE SEMENTES DE URUCUM EM LEITO DE JORRO
}

\author{
C. P. COELHO ${ }^{1}$, E. B. SANTANA ${ }^{2}$, L. J. G. FARIA ${ }^{3}$, C. M. L. COSTA ${ }^{3}$
}

${ }^{1}$ Bolsista de Iniciação Científica - PIBIC, Universidade Federal do Pará

${ }^{2}$ Doutoranda do Programa de Pós-Graduação em Recursos Naturais da Amazônia - PRODERNAUniversidade Federal do Pará

${ }^{3}$ Docente, Faculdade de Engenharia Química, ITEC - Universidade Federal do Pará

E-mail para contato: cmlc@ufpa.br

\begin{abstract}
RESUMO - As sementes de urucum se destacam como uma importante fonte de corante natural encontrada no país e é justificada pela sua toxicidade nula. Este trabalho objetiva a caracterização física das sementes de urucum, o estudo do comportamento fluidodinâmico em leito de jorro nas cargas de $630 \mathrm{~g}$ e $1000 \mathrm{~g}$, determinando os parâmetros específicos experimentais como: queda de pressão máxima, queda de pressão no jorro estável, queda de pressão no jorro mínimo, velocidade de jorro mínimo, dentre outros. Estes parâmetros também foram estimados por correlações da literatura, onde se obteve valores de desvio aceitáveis de $11,08 \%$ e $22,83 \%$ para velocidade de mínimo jorro para as duas cargas, respectivamente.
\end{abstract}

\section{INTRODUÇÃO}

As sementes de urucum (Bixa Orellana L.) se destacam como uma importante matéria prima encontrada no país para a obtenção de corantes, devido principalmente às características de produto natural, não tóxico, elevado poder tintorial e amplo espectro de cores. O pigmento corante do urucum está localizado na superfície do pericarpo da semente, ou seja, na sua porção mais externa. É constituído por vários carotenóides, predominando a bixina, e se encontra aderido à superfície da semente por meio de uma resina (Faria, 1998).

Logo, a extração do corante por processo mecânico, ou seja, com base no atrito e impacto das partículas, pode ser uma boa alternativa ao invés de extração por solventes, não somente para minimizar a degradação térmica e oxidativa dos pigmentos como reduzir os resíduos oriundos do processamento por solvente. Assim, a técnica do leito de jorro surge como uma alternativa para a extração mecânica de corante de urucum por possibilitar operar com partículas grandes, favorecer o contato fluido-partícula e um movimento padrão cíclico que proporciona um controle mais efetivo do processo.

Entretanto, o jorro é um fenômeno dependente de várias combinações como velocidade do gás e características das partículas sólidas, por exemplo, o formato e as aspectos superficiais das partículas têm influência na estabilidade do jorro.

Desta forma, visando contribuir para o aproveitamento industrial da semente de sementes de urucum/extração de corante, o presente trabalho tem como objetivo a caracterização física das sementes de urucum e avaliar o processo fluidodinâmico em leito de jorro para as sementes de urucum. 


\section{MATERIAIS E MÉTODOS}

O trabalho foi conduzido no Laboratório de Caracterização de Partículas em conjunto com o Laboratório de Secagem e Recobrimento de Partículas, ambos da UFPA. A matéria prima utilizada foram sementes híbridas de urucum, cultivadas nos campos experimentais da EMBRAPA - Empresa Brasileira de Pesquisa Agropecuária (Embrapa) - PA.

\subsection{Caracterização Física e Fluidodinâmica em Leito de Jorro}

A caracterização física das sementes de urucum foi efetuada com base em métodos padronizados, determinando-se as seguintes propriedades físicas: Diâmetro médio $\left(\mathrm{d}_{\mathrm{p}}\right)$, determinado pela técnica de peneiramento (McCabe et al, 1993); massa específica aparente $\left(\rho_{\text {ap }}\right)$ e massa específica real $\left(\rho_{\text {real }}\right)$, determinadas através do método de picnometria de comparação com tolueno (Keey, 1992); esfericidade $(\varphi)$, determinada através da razão entre diâmetros dos círculos inscritos e circunscritos ao contorno da projeção da partícula (Peçanha e Massarani, 1986).

O processo fluidodinâmico das sementes de urucum foi realizado em um leito de jorro cone-cilíndrico com configuração tradicional, ângulo de $45^{\circ}, 14 \mathrm{~cm}$ de altura, diâmetro de entrada do ar de $2,54 \mathrm{~cm}$ e diâmetro superior de $16 \mathrm{~cm}$, confeccionado em resina acrílica. $\mathrm{O}$ corpo cilíndrico possui $16 \mathrm{~cm}$ de diâmetro interno e $80 \mathrm{~cm}$ de altura.

Na Tabela 1 estão sumarizadas as correlações utilizadas para as análises dos parâmetros fluidodinâmicos.

Tabela 1- Equações para os parâmetros fluidodinâmicos

\begin{tabular}{|c|c|c|c|}
\hline Parâmetro & Autor & Equação & \\
\hline \multirow{2}{*}{$\Delta \mathrm{P}_{\max }$} & $\begin{array}{c}\text { Markowski e } \\
\text { Kaminski } \\
(1983)\end{array}$ & $\frac{\Delta P_{\max }}{\rho_{b} g H}=1+6,65\left(A r^{-0,2}\right)\left[\left(\frac{H}{D_{i}}\right)^{1,2}\right]\left\{\operatorname{tg}\left(\frac{\theta}{2}\right)^{0,5}\right\}$ & (1) \\
\hline & $\begin{array}{l}\text { Olazar et al. } \\
\quad(1994)\end{array}$ & $\frac{\Delta P_{\max }}{\Delta P_{j e}}=1+0,166\left[\left(\frac{H}{D_{i}}\right){ }^{1,80}\right]\left\{\left[\operatorname{tg}\left(\frac{\theta}{2}\right)\right]^{-0,80}\right\}\left(A r^{0,0125}\right)$ & (2) \\
\hline \multirow{2}{*}{$\Delta \mathrm{P}_{\mathrm{je}}$} & $\begin{array}{l}\text { Olazar et al. } \\
\quad(1994)\end{array}$ & $\frac{\Delta P_{j e}}{\rho_{b} g H_{j e}}=0,04\left[\left(\frac{H_{j e}}{D_{i}}\right)^{1,10}\right]\left(R e_{j m, i}^{0,20}\right)\left\{\left[\operatorname{tg}\left(\frac{\theta}{2}\right)\right]^{-0,11}\right\}\left(A r^{0,0125}\right)$ & (3) \\
\hline & $\begin{array}{l}\text { Pallai e } \\
\text { Németh } \\
(1969)\end{array}$ & $\frac{\Delta P_{j e}}{\Delta P_{\max }}=0,8-\left[0,01\left(\frac{D_{c}}{D_{i}}\right)\right]$ & (4) \\
\hline \multirow[t]{2}{*}{$\Delta \mathrm{P}_{\mathrm{jm}}$} & $\begin{array}{l}\text { Mujumdar } \\
\text { (1981) }\end{array}$ & $\frac{\Delta P_{j m}}{\rho_{p}\left(U i_{j m}\right)^{2}}=2,35(A r)\left(\frac{H}{d_{p}}\right) R e i_{j m}^{-2,285}$ & (5) \\
\hline & $\begin{array}{l}\text { Nascimento } \\
\text { et al. (1976) }\end{array}$ & $\Delta P_{j m}=\frac{2}{3}\left(1-\varepsilon_{m f}\right) H_{j m} \rho_{p} g$ & (6) \\
\hline \multirow[t]{2}{*}{$\mathrm{U}_{\mathrm{jm}}$} & $\begin{array}{l}\text { San Jose } \\
(1996)\end{array}$ & $\left(R e_{i}\right)_{j m}=0,126 A r^{0,5}\left[\left(\frac{D_{b}}{D_{i}}\right) 1,68\right]\left\{\left[\operatorname{tg}\left(\frac{\theta}{2}\right)\right]^{-0,57}\right\}$ & (7) \\
\hline & $\begin{array}{l}\text { Olazar et al. } \\
\quad(1993)\end{array}$ & $\left(R e_{i}\right)_{j m}=0,174 A r^{0,5}\left[\left(\frac{D_{b}}{D_{i}}\right)^{0,85}\right]\left\{\left[\operatorname{tg}\left(\frac{\theta}{2}\right)\right]^{-1,25}\right\}$ & (8) \\
\hline
\end{tabular}


Foram realizados testes fluidodinâmicos com cargas operacionais de $630 \mathrm{~g}$ e $1000 \mathrm{~g}$ de sementes de urucum a temperatura ambiente $\left(25^{\circ} \mathrm{C}\right)$. A partir das curvas características foram estimados os parâmetros fluidodinâmicos do leito de jorro, como: queda de pressão máxima $\left(\Delta \mathrm{P}_{\max }\right)$, queda de pressão no jorro estável $\left(\Delta \mathrm{P}_{\mathrm{je}}\right)$, queda de pressão no jorro mínimo $\left(\Delta \mathrm{P}_{\mathrm{jm}}\right) \mathrm{e}$ velocidade de jorro mínimo $\left(\mathrm{U}_{\mathrm{jm}}\right)$.

\section{RESULTADOS E DISCUSSÃO}

\subsection{Caracterização das Partículas}

$\mathrm{Na}$ Tabela 2 são apresentados os resultados da caracterização física das sementes.

Tabela 2 - Resultados da caracterização física da semente de urucum

\begin{tabular}{c|c|c|c|c}
\hline Parâmetros & $\mathrm{d}_{\mathrm{p}}(\mathrm{mm})$ & $\rho_{\mathrm{ap}}\left(\mathrm{g} / \mathrm{cm}^{3}\right)$ & $\mathrm{P}_{\text {real }}\left(\mathrm{g} / \mathrm{cm}^{3}\right)$ & $\varphi(\operatorname{adim})$. \\
\hline $\begin{array}{c}\text { Valor médio } \pm \\
\text { erro padrão }\end{array}$ & $3,524 \pm 0,021$ & $0,631 \pm 0,007$ & $1,638 \pm 0,007$ & $0,817 \pm 0,011$ \\
\hline
\end{tabular}

Segundo Gomes (1990), os resultados mostrados na Tabela 2 apresentam boa precisão na determinação das propriedades físicas das sementes, por apresentarem baixos valores para o erro padrão da média.

Com base nas propriedades diâmetro médio e massa específica, apresentadas na Tabela 2 para a matéria prima em estudo, foi identificado o tipo de partícula que caracteriza as sementes de urucum. Verifica-se que as mesmas são classificadas como sendo partículas do grupo D, segundo Geldart (1973), indicando a utilização do leito de jorro para os processos em leito móvel com contato gás-sólido.

\subsection{Análise Fluidodinâmica}

Para realizar o estudo da fluidização e verificar as melhores condições de mistura para as sementes de urucum foram determinados os parâmetros fluidodinâmicos. Esses índices são geralmente considerados como indicativos do comportamento dinâmico em leito móvel e pode ser explorado como uma forma de monitorar estes processos. Assim, na Tabela 3 são sumarizados os valores dos parâmetros fluidodinâmicos, onde se observa um aumento no espaço de vazios, em função da carga de sólidos, fato este, que pode ser explicado pelo modo de empacotamento dos sólidos no leito.

Tabela 3 - Parâmetros fluidodinâmicos para as diferentes cargas

\begin{tabular}{ccccccc}
\hline $\begin{array}{c}\text { Carga } \\
(\mathrm{g})\end{array}$ & $\begin{array}{c}\varepsilon_{0} \\
(\text { adim. })\end{array}$ & $\begin{array}{c}\varepsilon_{\mathrm{jm}} \\
(\operatorname{adim} .)\end{array}$ & $\begin{array}{c}\mathrm{H}_{0} \\
(\mathrm{~cm})\end{array}$ & $\begin{array}{c}\mathrm{H}_{\mathrm{jm}} \\
(\mathrm{cm})\end{array}$ & $\begin{array}{c}\mathrm{E} \\
(\%)\end{array}$ & $\begin{array}{c}\mathrm{H}_{\mathrm{jm}} / \mathrm{H}_{0} \\
(\text { adim. })\end{array}$ \\
\hline 630 & 0,61 & 0,67 & 1,1 & 1,3 & 15,38 & 1,18 \\
1000 & 0,87 & 0,88 & 4,5 & 4,8 & 6,25 & 1,06 \\
\hline
\end{tabular}

Sobre a expansão (E) do leito, observou que houve uma diminuição da expansão inversamente ao aumento de carga, devido ao aumento das forças de coesão provocadas por essa elevação de massa. A Figura 1 também demonstra a influência da carga juntamente com 
a velocidade sobre o coeficiente de expansão $\left(\mathrm{H} / \mathrm{H}_{0}\right)$. Observa-se que mesmo sendo necessária a inserção de valores mais elevados de velocidade para a carga de $1000 \mathrm{~g}$, os valores obtidos para a menor carga foi superior a 1,18, comparada a de $630 \mathrm{~g}$ que foi de 1,06; comportamento também observado por Basu Paudel (2011).

Figura 1 -Comportamento do coeficiente de expansão versus velocidade de escoamento.

Figura 1.a - Carga de $630 \mathrm{~g}$.

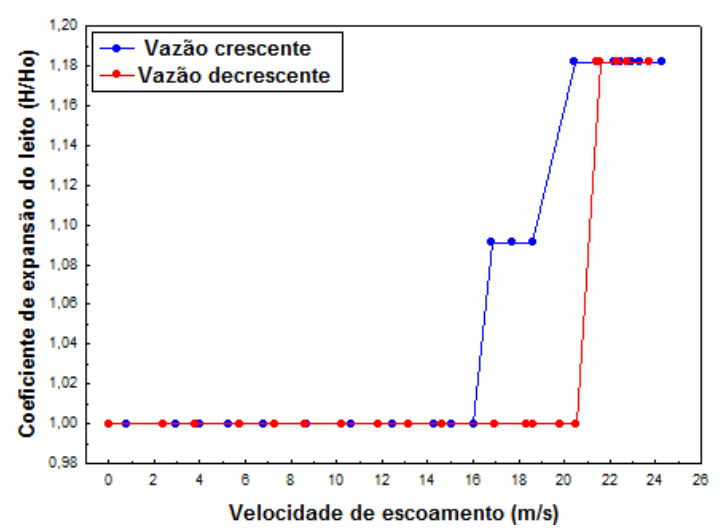

Figura 1.b - Carga de $1000 \mathrm{~g}$.

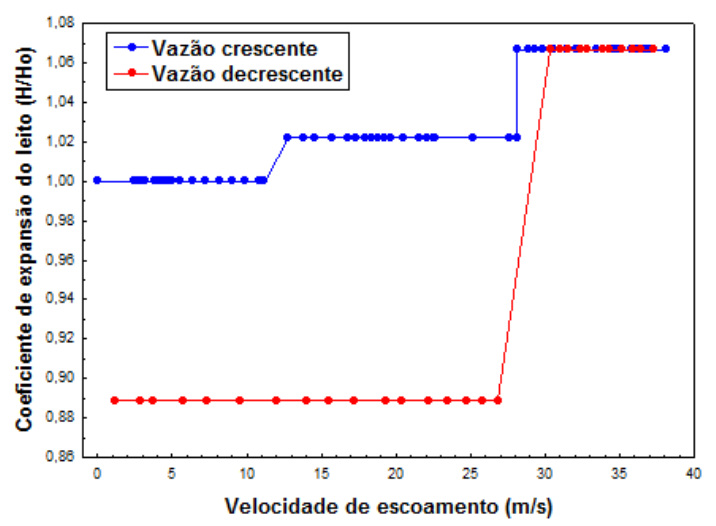

Observa-se também, que a vazão decrescente proporcionou para a carga menor o retorno a sua altura inicial, este fato deve ter sido obtido devido ao menor número de partículas que se compactaram em seu estado inicial, comportamento não reproduzido para a maior carga.

A Figura 2 e a Tabela 4 mostram que o aumento de carga teve influência sobre os parâmetros fluidodinâmicos, como é verificado na velocidade de mínimo jorro com valor de $21,58 \mathrm{~m} / \mathrm{s}$ para a carga de $630 \mathrm{~g}$ e de 31,06 para a carga de $1000 \mathrm{~g}$. Tal comportamento também é observado por Almeida (2002) e Santana (2011), que avaliaram a fluidodinâmica em leito de jorro de sólidos como sementes de brócolos e sementes de linhaça, respectivamente.

Figura 2 - Curvas fluidodinâmicas características.

Figura 2.a - Carga de $630 \mathrm{~g}$.

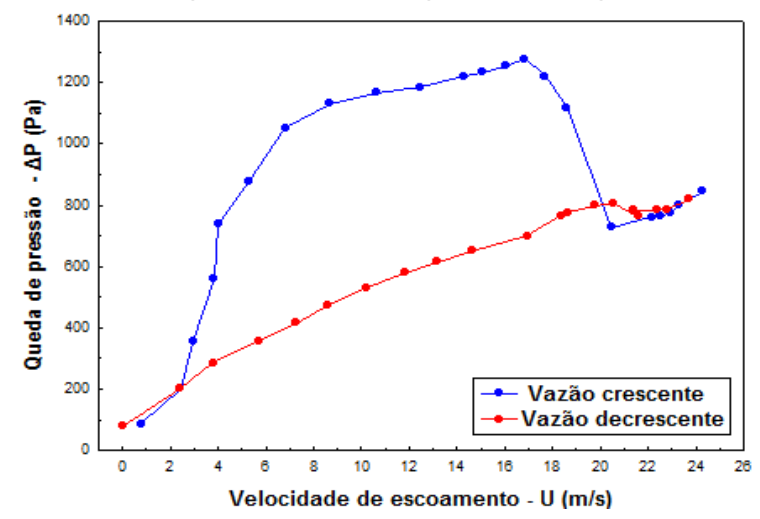

Figura 2.b - Carga de $1000 \mathrm{~g}$.

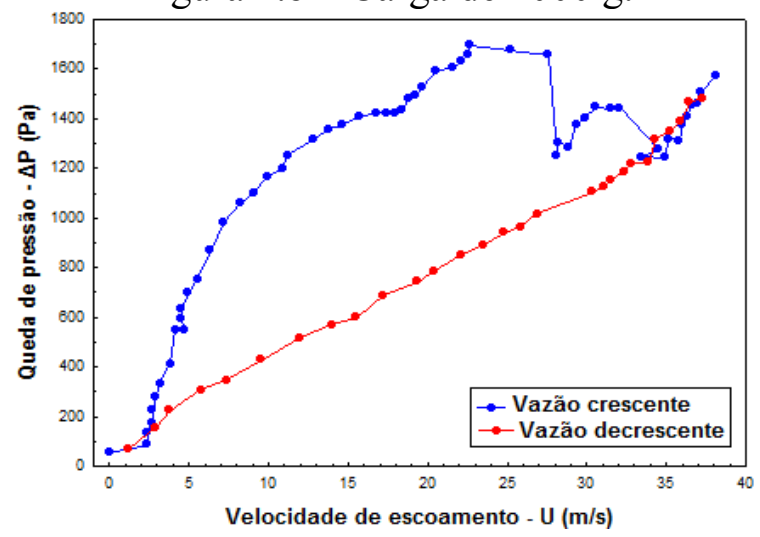


A análise do comportamento fluidodinâmico em relação à queda de pressão máxima não apresentou desvios aceitáveis para carga de $630 \mathrm{~g}$ para nenhuma das correlações avaliadas. Entretanto, a equação de Markowski e Kaminski (1983) apresentou resultado aceitável para carga de $1000 \mathrm{~g}$ com desvio de 9,49\%.

Tabela 4 - Análise dos parâmetros fluidodinâmicos

\begin{tabular}{|c|c|c|c|c|c|}
\hline \multirow{3}{*}{$\begin{array}{l}\text { Parâmetro/ } \\
\text { Unidade }\end{array}$} & \multirow{3}{*}{ Dados } & \multicolumn{4}{|c|}{ Carga/Unidade } \\
\hline & & \multicolumn{2}{|c|}{$630 \mathrm{~g}$} & \multicolumn{2}{|c|}{$1000 \mathrm{~g}$} \\
\hline & & Valores & Desvios $(\%)$ & Valores & Desvios $(\%)$ \\
\hline \multirow{3}{*}{$\Delta \mathrm{P}_{\max }(\mathrm{Pa})$} & Exp. & 1276,42 & ---- & 1697,39 & ---- \\
\hline & Eq. 1 & 225,75 & 82,31 & 1858,48 & 9,49 \\
\hline & Eq. 2 & 778,38 & 54,55 & 1199,38 & 29,34 \\
\hline \multirow{3}{*}{$\Delta \mathrm{P}_{\mathrm{je}}(\mathrm{Pa})$} & Exp. & 762,32 & ----- & 1243,87 & ----- \\
\hline & Eq. 3 & 87,30 & 80,55 & 729,79 & 41,33 \\
\hline & Eq. 4 & 467,89 & 38,62 & 528,83 & 57,48 \\
\hline \multirow{3}{*}{$\Delta \mathrm{P}_{\mathrm{jm}}(\mathrm{Pa})$} & Exp. & 762,06 & ----- & 1125,99 & ----- \\
\hline & Eq. 5 & 672,68 & 11,73 & 3575,09 & 217,51 \\
\hline & Eq. 6 & 181,65 & 76,6 & 78,88 & 92,99 \\
\hline \multirow{3}{*}{$\mathrm{U}_{\mathrm{jm}}(\mathrm{m} / \mathrm{s})$} & Exp. & 21,58 & ----- & 31,06 & ------ \\
\hline & Eq. 7 & 23,97 & 11,08 & 23,97 & 22,83 \\
\hline & Eq. 8 & 15,24 & 29,36 & 15,24 & 50,92 \\
\hline
\end{tabular}

Para os valores da queda de pressão no jorro mínimo obtidos experimentalmente apenas a correlação Mujumdar (1981) apresentou um valor considerado aceitável, ou seja, inferior a $20 \%$ na ordem 11,73 \% para a carga de 630 g. Os menores desvios para o parâmetro velocidade de mínimo jorro são obtidos pela correlação de San Jose (1996), para ambas as cargas, na ordem de 11,08 e $22,83 \%$, considerando que para carga de $1000 \mathrm{~g}(22,83 \%)$, ainda pode se considerar aceitável, uma vez que o valor é numericamente próximo da região considerada aceitável.

\section{REFERÊNCIAS BIBLIOGRÁFICAS}

ALMEIDA, C.; ROCHA, S. C. S. Fluidodinâmica de sementes de brócolos em leito fluidizado e leito de jorro. Scientia Agricola, v. 59, n. 4, p. 645-652, 2002.

BASU PAUDEL, B. E. Experimental study on fluidization of biomass, inert particles, and biomass/sand mixtures. p. 77, Thesis Prepared for the Degree of Master of Science University of North Texas, 2011.

FARIA, L. J. G. Análise experimental do processo de secagem de urucum (Bixa Orellana L.) em leito fixo. Campinas: 1998. 252 f. Tese (Doutorado em Engenharia Química) Faculdade de Engenharia Química, Universidade Estadual de Campinas-SP, p. 1-162. 


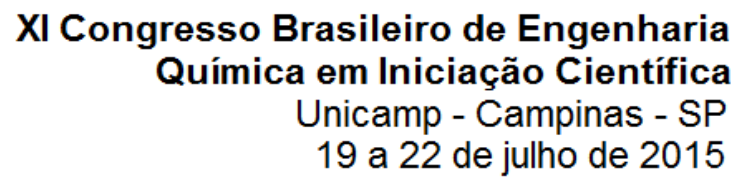

GOMES, F. P. A Estatística Moderna na Pesquisa Agropecuária. 3. ed. Piracicaba: Nobel, 1987.

KEEY, R. B. Drying of loose and particulate materials. New York: Hemisphere Publishing, 1992.

MARKOWSKI, A.; KAMINSKI, W. Hydrodynamic Characteristics of Jet-Spouted Beds, The Canadian Journal of Chemical Engineering, v.61, p. 377-381, 1983.

MATHUR, K. B.; GISHLER, P. E. A technique for contacting gases with coarse solid particles. A.ICh.E.J., n.1, p.157, 1954.

McCabe, W.L.; SMITH, J. C.; HARRIOTT, P. Unit operations of chemical engineering, 5 ed., New York: McGraw-Hill, 1993.

MUJUMDAR, A. S. Drying of granular materials, lecture notes. Montreal: Departament of Chemical Engineering, McGill University, 1981.

OLAZAR, M.; SAN JOSÉ, M. J.; AGUAYO, A. T.; BILBAO, J. Hydrodynamics of nearly flat base spouted beds. Chemical Engineering Journal, v.55, p.27-37, 1994.

OLAZAR, M.; SAN JOSÉ, M. J.; PEÑAS, F. J.;AGUAYO, A. T.; BILBAO, J. Stability and hydrodynamics of conical spouted beds with binary mixtures. Ind. Eng. Chem. Res. p. 2826-2835, 1993.

PALLAI, I.; NEMETH, J. Analysis of flow in a spouted bed apparatus by the so called phase diagram. In: International Congress Chemical Engineer (CHISA), 1969.

PEÇANHA, R. P.; MASSARANI, G. Dimensão característica e forma das partículas. In: XIV Encontro sobre Escoamento em Meios Porosos (XIV ENEMP). 1986, Campinas. Anais... Campinas-SP: FEQ/ UNICAMP, 1986, p. 302-312.

SAN JOSE, M. J.; OLAZAR, M.; AGUADO, R. E.; BILBAO, J. Influence of the conical section geometry on the hydrodynamics of shallow spouted. Chemical Engineer Journal, v.62, p.113-120, 1996.

SANTANA, E. B. Análise experimental do comportamento fluidodinâmico e da secagem de sementes de linhaça (Linum usitatissimum L.) em leito de jorro. Belém: 2011. $142 \mathrm{f}$. Dissertação (Mestrado em Engenharia Química) - Instituto de Tecnologia, Universidade Federal do Pará, p.76. 